\title{
Phonon Effects on Spin-Charge Separation in One Dimension
}

\author{
Wen-Qiang Ning, ${ }^{1,2}$ Hui Zhao, ${ }^{1}$ Chang-Qin Wu, ${ }^{1, *}$ and Hai-Qing Lin $^{2,1}$ \\ ${ }^{1}$ Department of Physics, Fudan University, Shanghai 200433, China \\ ${ }^{2}$ Department of Physics and Institute of Theoretical Physics, \\ The Chinese University of Hong Kong, Shatin, Hong Kong, China
}

(Dated: September 13, 2018)

\begin{abstract}
Phonon effects on spin-charge separation in one dimension are investigated through the calculation of one-electron spectral functions in terms of the recently developed cluster perturbation theory together with an optimized phonon approach. It is found that the retardation effect due to the finiteness of phonon frequency suppresses the spin-charge separation and eventually makes it invisible in the spectral function. By comparing our results with experimental data of TTF-TCNQ, it is observed that the electron-phonon interaction must be taken into account when interpreting the ARPES data.

PACS numbers: 63.20.Kr, 71.27.+a, 71.30.+h
\end{abstract}

It is commonly accepted that most one-dimensional (1D) correlated electronic systems cannot be properly described by the traditional Fermi liquid theory, instead, their behaviors are well predicted by the Luttinger liquid theory [1, 2, 3, 4]. One of the key features of the Luttinger liquid is the spin-charge separation: the lowenergy excitations are not quasiparticles with charge $e$ and spin $1 / 2$ together, rather, they are collective modes of spin and charge excitations separately, called spinons and holons. Since spinon and holon move with different speed, they eventually decouple. Following earlier works [1, 2, 3, 4], many studied spin-charge separation with various theoretical schemes. In particular, one could explore the existence of the spin-charge separation by calculating the spectral function $5,6,6,8,9,10$, which has direct relation to the angle-resolved photoemission spectroscopy (ARPES). In some recent works, the spinon and holon branches have been observed by ARPES performed on some 1D materials such as $\mathrm{SrCuO}_{2}$. 11, 12, 13, 14] Because both electron-electron and electron-phonon interactions exist in many low-dimensional materials, it is important to address the role of these interactions on the spin-charge separation. 6, 15, 16, 17, 18, 19.

The one-dimensional Holstein-Hubbard model (HHM), which is the simplest model involving both electronphonon (e-p) and electron-electron (e-e) interactions, has been used extensively to describe some low-dimensional materials. Since electrons in these materials are strongly correlated, the interplay between electron-phonon interaction and Coulomb repulsion should have profound effect on the spin-charge separation, and we expect to observe these effects by investigating the single-particle excitation spectra. The spectral function provides valuable insights into the usually complicated many-body systems, such as high-temperature superconductors, cuprate ladder compounds, and organic conductors. For example, very recently, by using the exact diagonalization method, Fehske et al. 20 calculated the spectral function of the Holstein-Hubbard model on a finite system and found a
Mott-insulator to Peierls-insulator transition at a compatible ratio of the e-e to e-p interactions.

Bearing these in mind, we compute the one-electron spectral function of the HHM by applying the recently developed cluster perturbation theory (CPT) $8,21,22$ together with an optimized phonon approach. 23, 24, 25] The spectral function at full frequency region with rich satellite structures is obtained in the model of both e-e and e-p interactions for the first time. Phonon effects on spin-charge separation are focused in the presence of e-e interactions from weak to strong coupling. It is found that the retardation effect due to the finiteness of phonon frequency does not favor the spin-charge separation. In weak interaction regimes, a peak in the spectral function was observed which is consistent with the existence of a metallic phase as proposed recently by Clay and Hardikar 27]. Furthermore, it is observed that one must take electron-phonon interaction into account when interpreting the ARPES experimental data in the one dimensional material.

The HHM accounts for a tight-binding electron band, on-site Coulomb repulsion between electrons of opposite spin, and coupling of charge carriers to local phonons:

$$
\begin{aligned}
H= & -t \sum_{i, \sigma}\left(c_{i, \sigma}^{\dagger} c_{i+1, \sigma}+H . c .\right)+U \sum_{i} n_{i \uparrow} n_{i \downarrow} \\
& -g \sum_{i, \sigma}\left(b_{i}^{\dagger}+b_{i}\right) n_{i, \sigma}+\omega_{0} \sum_{i} b_{i}^{\dagger} b_{i},
\end{aligned}
$$

where $c_{i, \sigma}^{\dagger}\left(c_{i, \sigma}\right)$ creates (annihilates) an electron with $\sigma$ on site $i$, and $b_{i}^{\dagger}$ and $b_{i}$ are creation and annihilation operators of the local phonon mode at site $i$, respectively. $t$ is the electron hopping constant between nearest neighbor sites which will be set as the energy unit in our calculations, $\omega_{0}$ is the bare phonon frequency, and $g$ is the electron-phonon coupling constant.

For the calculation of the spectral properties within the framework of CPT [8, 21], one divides the lattice into fully equivalent clusters of a finite sites. For each cluster, 
we calculate the Green's function $G_{i, j}(z)\left(\equiv G_{i, j}^{+}(z)+\right.$ $\left.G_{i, j}^{-}(z)\right)$, with $G_{i, j}^{ \pm}(z)$ defined as

$$
G_{i, j}^{ \pm}(z)=\left\langle\phi_{0}\left|c_{i}^{ \pm} \frac{1}{z \pm\left(H-E_{0}\right)} c_{j}^{\mp}\right| \phi_{0}\right\rangle,
$$

where $c_{i}^{+} \equiv c_{i}^{\dagger}, c_{i}^{-} \equiv c_{i}$, and $\left|\phi_{0}\right\rangle$ being the ground state of the cluster, which is obtained by using the Lanczos exact diagonalization (ED) method within an optimized phonon approach 24, 25] under open boundary conditions. Two terms in $G_{i, j}$ corresponding to electron and hole propagation, respectively, can be obtained. The CPT treats the intercluster hopping by a strong-coupling perturbation, i.e., $(t / U)$ expansion. The lowest-order CPT approximation to Green's function gives

$$
G_{C P T}(k, z)=\frac{1}{N} \sum_{i, j} e^{-i k(i-j)} \widetilde{G}_{i, j}(N k, z),
$$

where $\widetilde{G}_{i, j}(Q, z)$ is the Green's function of the full system and $N$ is the size of clusters. The spectral function is then $A(k, \omega)=-\frac{1}{\pi} \operatorname{Im}\left[G_{C P T}(k, z)\right]$, where $z=\omega+i \eta$ with $\eta$ defines the width of peaks in the spectral function. Since the Fermi energy is set to zero, the spectral function has the symmetry of $A(k, \omega)=A(\pi-k,-\omega)$ due to the electron-hole symmetry of the model (1).

In the absence of interactions, $A(k, \omega)$ obtained by the CPT method is exact [8, 21], while for interacting models, the accuracy of CPT depends on the size of the cluster and the number of optimal phonon chosen. To test the accuracy of the approach we use, we calculated the spectral function of the Hubbard model with exactly the same parameters as used by Benthien et al [9] and obtained agreeable results. We also calculated the first two spectral momenta and they match exactly to those obtained by White 26. Furthermore, based on our previous technique analysis 23], system parameters were carefully chosen in this work to ensure that our results mimic thermodynamic limit. Results obtained in this Letter were for $N=6, \eta=0.1 t$, and three optimized phonons at each site, with relative error $10^{-5}$ for the total energy. 23.

Three energy scales govern the physics of the HHM: the on-site Coulomb repulsion $(U)$, the electron-phonon coupling $\left(\lambda=2 g^{2} / \omega_{0}\right)$ and the bare phonon frequency $\left(\omega_{0}\right)$. The ground state of the system at half-filling is a Mott-Hubbard insulating (MI) state when $U$ is large, and shows spin-density-wave (SDW) fluctuations. When electron-phonon interaction dominates, the system is in the Peierls insulating state (PI), characterized by the charge-density-wave where both spin gap and change gap are finite, while in the MI state, the spin gap vanishes. Very recently, it was reported that there is a metallic region intermediate between the PI and MI states with superconducting pairing correlation dominates. 27.

In the absence of phonons $(g=0)$, Eq. (II) is just the Hubbard model whose physics have been extensively

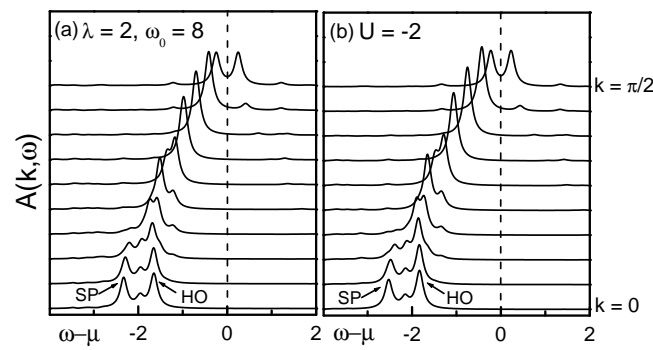

FIG. 1: The obtained spectral function $A(k, \omega)$ of (a) the large- $\omega_{0}$ Holstein model and (b) the negative-U Hubbard model at half-filling. HO and SP stand for holon and spinon excitations respectively.

studied and it is well known that in the Hubbard model, spin and charge separate. 7, 8, 9, 10] On the other hand, when $U=0$, Eq. (1) is another extensively studied model, the Holstein model (HM). In the strong electronphonon coupling region, the ground state of the Holstein model at half filling is either a bipolaron insulating (BPI) state in the large $\omega_{0}$ limit, characterized by the configuration where each site is either empty or doubly occupied because phonons produce an attraction between the electrons, or a traditional band insulating state in the small $\omega_{0}$ limit. 20, 28] In the weak coupling region, the Peierls gap is suppressed by the phonon quantum fluctuations and the ground state is at metallic (M) phase. 23, 24, 29. When the phonon degrees of freedom are integrated out, the spin-1/2 Holstein model could be mapped onto the Hubbard model with an effective dynamical attraction $U_{\text {eff }}(\omega)=-\lambda /\left(1-\omega^{2} / \omega_{0}^{2}\right)$. Here one also expects to observe the spin-charge separation. In the anti-adiabatic limit, i.e., $\omega_{0} \rightarrow \infty$, the attraction becomes instantaneous and equals to the bipolaron binding energy $\lambda$, which has already been reported long time ago. 28] Obviously, for any finite phonon frequency $\omega_{0}$, one must consider the retardation effect fully which could not be simply presented by the above $U_{\text {eff }}(\omega)$.

To have a sense of the magnitude of $\omega_{0}$, we give a comparison of the spectral function of these two models in Figure 1. It is clearly shown that the spectral function of the Holstein model is almost the same as that of the negative-U Hubbard model. Some minor differences due to finiteness of $\omega_{0}$ are invisible in the figure (in other words, $\omega_{0}=8$ is almost at the antiadibatic limit). This result is not trivial as seen at first glance because it implies the single-particle excitation of the system with a large phonon frequency is similar to that at the antiadiabatic limit, which is consistent with the existence of a quantum metal-insulator phase transition in the Holstein model. 27, 29, 30] The peaks labelled "SP" and "HO" in Fig. 1 refer to the spinon and the holon branches, respectively, signaturing the spin-charge separation. Compare to the conventional Luttinger liquids (e.g., the positive-U Hubbard model), the charge velocity $\left(v_{\rho}\right)$ is smaller than 


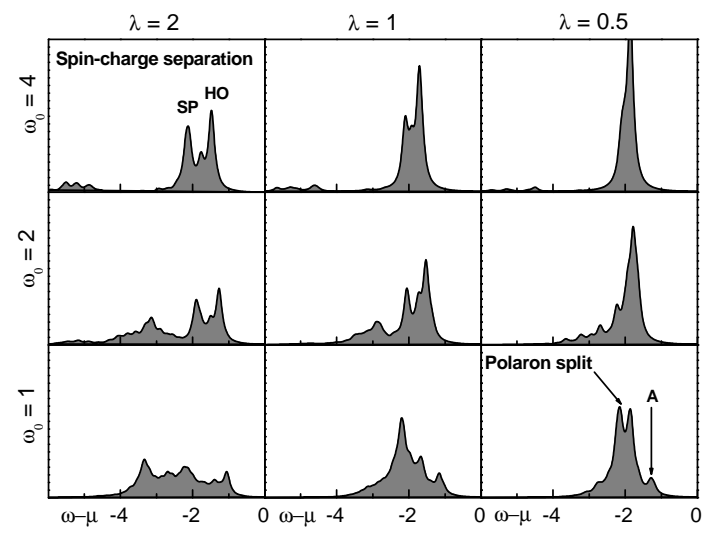

FIG. 2: The spectral function $A(0, \omega)$ of the Holstein model.

the spin velocity $\left(v_{\sigma}\right)$.

Fig. 2 illustrates the retardation effects systematically. Starting from the strong coupling case $(\lambda=2)$, we observe that as we decrease $\omega_{0}$ from 4 to 2 , the incoherent part of the spectra becomes more important. As a consequence, the spectral weights of the spinon and holon excitations are much smaller. This could be regarded as a continuation from Fig. 1: $\omega_{0}=\infty \rightarrow 8 \rightarrow 4$. When the phonon frequency is further reduced, the spectral weights correspond to phonon excitations become dominant, which is quite different from that in the antiadiabatic regime where the "spinon" and "holon" excitations are clearly the dominant ones. Therefore, due to the strong mixing of the coherent and incoherent excitations, it is difficult to single out the spinon and holon excitations, instead, one observes an almost flat band dispersion with exponentially small spectral weight. The dominant peaks in the incoherent part of the spectra are related to multiples of the (large) bare phonon frequency broadened by electronic excitations. Such electron-phonon mixed nature of excitations could be seen in the spectra away from the Fermi surface.

It is quite natural to expect that the separation of spin and charge excitation will become smaller with the decrease of the electron-phonon coupling strength. This is clearly reflected in the spectral function. As shown in the first row of Fig. 2, when we reduce the electronphonon coupling $\lambda$ at $\omega_{0}=4$, the difference between the spinon and the holon excitation at $k=0$ becomes smaller and eventually invisible. As we reduce phonon frequency, retardation comes into play. It is also interesting to observe that there is an excitation split in the weak coupling case (Fig. 2, $\lambda=0.5, \omega_{0}=1$ ). Such splitting is not due to spin-charge separation. In fact, by carefully comparing this spectral function with that of a spinless Holstein model at corresponding electronphonon coupling, we found that the splitting is caused by the polaron interaction.

Figure 3 shows the spectra of up-spin electron for the

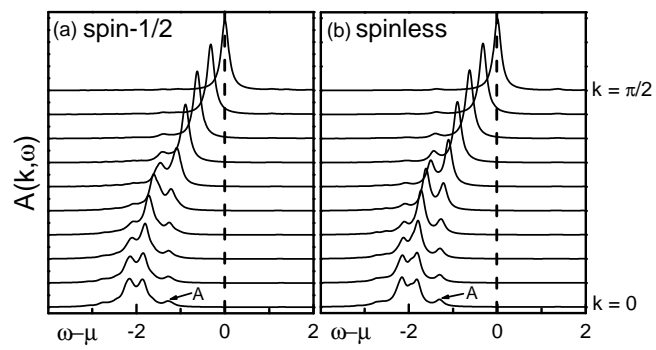

FIG. 3: Spectral function $A(k, \omega)$ of the spinful (a) and spinless (b) Holstein model. $\lambda=0.5$ and $\omega_{0}=1$.

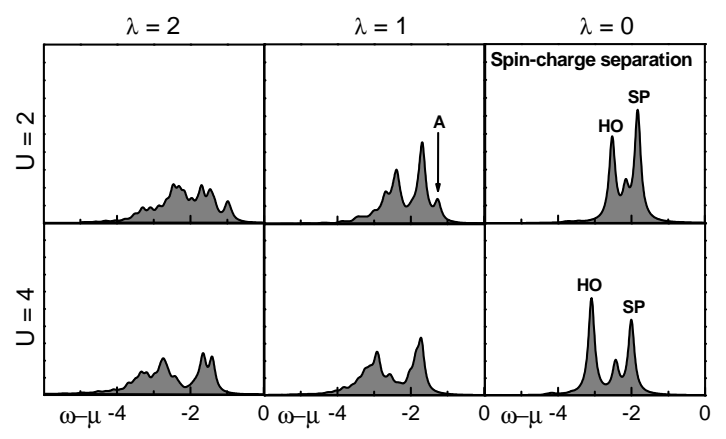

FIG. 4: Spectral function $A(0, \omega)$ of the Holstein-Hubbard model at half filling. $\omega_{0}=1$.

spin- $1 / 2$ Holstein model at half-filling in comparison with the spinless Holstein model. In the weak coupling regime the two spectral are almost the same, indicating that the existence of the down-spin electrons have nearly zero effect on the spectral function of the up-spin electrons. It shows that the phonon-mediated interaction between upand down-spin electrons is very weak so they are almost decoupled in this case. Thus the splitting of the excitations cannot be attributed to the spin-charge separation, rather, it is due to the interaction among polarons. Notice that there is a small peak labelled as "A" in the spectral weight, which is almost dispersionless in small $k$ regime (see Fig. 3) and is suppressed by the on-site repulsion $U$ (see Fig. 4). Since it is appeared in the metallic region intermediate between PI and MI phases, we speculate the peak "A" may be related with the electron pairing, as discussed recently by Clay and Hardiker 27, although further investigations are definitely deserved.

Now we turn the Coulomb interaction $U$ on and discuss its effect on the spectral function in the presence of electron-phonon interaction. From Figure 4, we also see that, the electron-electron interaction and the electron-phonon interaction have opposite effect on the spin-charge separation, as we intuitively expected. At given electron-electron interaction (for example, $U=$ 4 ), increasing the electron-phonon interaction tends to broaden the excitation bands and leave the spin-charge separation invisible. While at fix electron-phonon interaction, the electron-electron interaction increases the sep- 


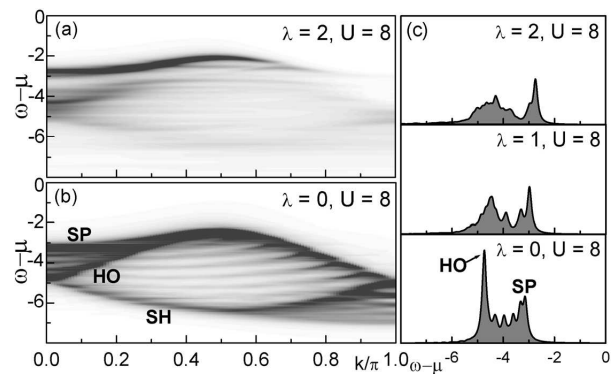

FIG. 5: Density plot of the spectral function $A(k, \omega)$ for (a) the Holstein-Hubbard, and (b) the Hubbard model. (c) The spectral function $A(0, \omega)$ of corresponding models at different electron-phonon coupling. SH stands for the shadow band. $\omega_{0}=1$.

aration between the spin and the charge excitations.

By further increasing the electron-electron interaction, we illustrate the role of electron-phonon coupling in Fig. 5. Comparing Fig. 5(b) with Fig. 5(a), one may still observe the signature of spin-charge separation as $\lambda$ increases, but the holon branch is much more broadened and its spectral weight decreases, while the spinon branch is nearly unchanged. This is clearly shown in Fig. 5(c). Consequently, the so-called shadow band, which originates from the diverging spin fluctuations at $2 k_{F}, 8,31,32,33$ is nearly gone, due to the fact that the shadow band is actually the continuation of the holon band.

Finally, we make a comparison on our results to the ARPES experiments of the quasi-one-dimensional organic conductor TTF-TCNQ. 13, 14] We notice that in the Fig. 7 of the Ref. 14], the charge branch (b) and the spin branch (a) are weakly connected with each other. The result can not be explained by the Hubbard model alone(Fig. 5(b)). As our results suggest, the electron-phonon interaction must be taken into account. From Fig. 5(a), it is clearly seen that the dispersion of the charge branch is weakened in the spectral function and the spinon branch is weakly connected to the holon branch. A similar broadening due to phonon is also observed in one dimensional $\mathrm{SrCuO}_{2}$. 34 These facts indicate that one should expect significant contribution from the electron-phonon interaction to the spectra of these strongly correlated quasi-one-dimensional materials. Of course, our conclusions are based on numerical studies of Eq. (1) so to have detailed analyzes of experiments, one may use models different from Eq. (1) but we believe essential physics remain unchanged.

In summary, by applying the CPT together with an optimized phonon approach, we have studied the spectral function of the one-dimensional Holstein-Hubbard model at half filling. A comprehensive picture for the spectral function in the presence of electron-electron interaction and electron-phonon interaction was presented. In particular, we addressed the issue of spin-charge sepa- ration and found that the electron-electron interaction competes with the electron-phonon interaction on the spin-charge separation, and the retardation effect due to phonons may diminish the spin-charge separation in the spectral function. We also found polaron splitting and observed a peak that may related to electron pairing in the weak e-p coupling limit.

This work was partially supported by the National Natural Science Foundation of China, CUHK 401504, and MOE B06011. The authors are grateful to Prof. R. B. Tao for his generous support and to Prof. D. L. Feng for helpful discussion on the ARPES data. We also thank Profs. H. Chen and H. Zheng for stimulating discussions and Prof. Z.-X. Shen for his preprint.

* Corresponding author. Email: cqw@fudan.edu.cn

[1] S. Tomonaga, Prog. Theor. Phys. 5, 544 (1950); J. M. Luttinger, J. Math. Phys. 4, 1154 (1963).

[2] E. H. Lieb and F. Y. Wu, Phys. Rev. Lett. 20, 1445 (1968).

[3] V. J. Emery, pp. 247-303 in Highly Conducting OneDimensional Solids, edited by J. T. Devreese et al. (Plenum, New York, 1979).

[4] F. D. M. Haldane, Phys. Rev. Lett. 45, 1358 (1980).

[5] V. Meden and K. Schönhammer, Phys. Rev. B 46, 15753 (1992); J. Voit, Phys. Rev. B 47, 6740 (1993).

[6] V. Meden et al., Phys. Rev. B 50, 11179 (1994).

[7] M. G. Zacher et al., Phys. Rev. B 57, 6370 (1998).

[8] D. Sénéchal et al., Phys. Rev. Lett. 84, 522 (2000).

[9] H. Benthien et al., Phys. Rev. Lett. 92, 256401 (2004).

[10] H. Matsueda et al., cond-mat 0505670 (unpublished).

[11] C. Kim et al., Phys. Rev. Lett. 77, 4054 (1996); C. Kim et al., Phys. Rev. B 56, 15589 (1997).

[12] K. Kobayashi et al., Phys. Rev. Lett. 82, 803 (1999).

[13] R. Claessen et al., Phys. Rev. Lett. 88, 096402 (2002).

[14] M. Sing et al., Phys. Rev. B 68, 125111 (2003).

[15] K. Tsutsui et al., Physica C 392-396, 199 (2003).

[16] I. P. Bindloss and S. A. Kivelson, Phys. Rev. B 71, 014524 (2005).

[17] L. Perfetti et al., Phys. Rev. B 66, 075107 (2002).

[18] D. S. Dessau et al., Phys. Rev. Lett. 81, 192 (1998).

[19] K. M. Shen et al., Phys. Rev. Lett. 93, 267002 (2004).

[20] H. Fehske et al., Phys. Rev. B 69, 165115 (2004).

[21] D. Sénéchal et al., Phys. Rev. B 66, 075129 (2002).

[22] M. Hohenadler et al., Phys. Rev. B 68, 184304 (2003).

[23] H. Zhao et al., Phys. Rev. B 71, 115201 (2005).

[24] C. Zhang et al., Phys. Rev. Lett. 80, 2661 (1998); Phys. Rev. B 60, 14092 (1999).

[25] A. Weisse et al., Phys. Rev. B 62, R747 (2000).

[26] S. R. White, Phys. Rev. B 44, 4670 (1991).

[27] R. T. Clay and R. P. Hardikar, Phys. Rev. Lett. 95, 096401 (2005).

[28] J. E. Hirsch and E. Fradkin, Phys. Rev. B 27, 4302 (1983).

[29] C. Q. Wu et al., Phys. Rev. B 52, R15683 (1995).

[30] E. Jeckelmann et al., Phys. Rev. B 60, 7950 (1999)

[31] S. Haas and E. Dagotto, Phys. Rev. B 52, R14396 (1995).

[32] K. Penc et al., Phys. Rev. Lett. 77, 1390 (1996). 
[33] J. Favand et al., Phys. Rev. B 55, R4859 (1997).

[34] B. J. Kim et al., preprint. 